\title{
A New Phase-Correlation Based Gradient Registration Approach for Phase-retrieval with DIC and DPC
}

\author{
Shalin B. Mehta, ${ }^{1,2,3, *}$ and Colin J. R. Sheppard ${ }^{1,2,4}$ \\ ${ }^{1}$ Optical Bioimaging Lab, Division of Bioengineering, National University of Singapore, \\ Block-E3A, \#7-10, 7 Engineering Drive 1, Singapore 117574 \\ ${ }^{2}$ NUS Graduate School for Integrative Sciences E Engineering (NGS), National University of Singapore \\ ${ }^{4}$ Department of Biological Sciences, National University of Singapore \\ *Corresponding author: shalin@nus.edu.sg,shalin.mehta@gmail.com
}

Phase-correlation robustly detects translations and rotations between images. We extend it for registration of images acquired using varied settings of differential interference contrast (DIC) and differential phase contrast (DPC) for phase-retrieval.

OCIS codes: (100.5070) Phase-retrieval, (100.4994) Pattern recognition, image transforms (C) 2009 Optical Society of America

Full-field phase-gradient contrast methods such as differential interference contrast (DIC) [1] and asymmetric illumination-based differential phase contrast (AIDPC) [2-4] allow imaging with large illumination apertures, resulting in high resolution, speckle-free images with depth sectioning. However, characteristic two-sided intensity variation of phase-gradient images makes image processing tasks such as feature segmentation difficult to perform. To allow computer-assisted processing of phase information, approaches for retrieval of phase information from at least two phase-gradients measured in orthogonal directions have been proposed [5,6]. One of the experimental difficulties in phase-retrieval process is to align phasegradient images taken at different bias settings of DIC or with different directions of illumination in AIDPC. The mis-registration occurs due to mechanical movement during acquisition. A noise-proof method for solving this problem does not seem to be available in the literature. We propose a novel approach that extends phase-correlation algorithm to registration of phase-gradient images, acquired along the same or the orthogonal directions of differentiation.

Among automated image registration methods, phase correlation $[7,8]$ is known to be robust against variety of noise sources, including additive noises and partial overlap. Phase-correlation relies on shift theorem of the Fourier transform, and involves finding a positive correlation peak in the normalized crossspectrum of the images to be registered. If $I_{t}$ and $I_{r}$ are target and reference images respectively, phasecorrelation of $I_{t}$ and $I_{r}$ is computed as,

$$
P\left(I_{t}, I_{r}\right)=\mathcal{F}^{-1}\left[\frac{\mathcal{F}\left(I_{t}\right) \mathcal{F}^{*}\left(I_{r}\right)}{\left|\mathcal{F}\left(I_{t}\right)\right|\left|\mathcal{F}\left(I_{r}\right)\right|}\right] .
$$

The relative shift of $I_{t}$ with respect to $I_{r}$ is given by, $(\Delta x, \Delta y)=\arg \max P\left(I_{t}, I_{r}\right)$. Although, it has been used with DIC, it has been found to work in some cases [6] and not in other cases [9]. In our experience, the phase-correlation approach of eq. 1 does not usually work with DIC images. The reason for this is evident when we realize that phase-correlation method is designed for registering two noisy and shifted measurements of the same two-dimensional function. In the case of DIC or AIDPC, we instead are registering two different functions, albeit related to the underlying phase of the specimen.

As can be seen from the references, phase-retrieval in both DIC and AIDPC requires computation of a ratio of images that represents linear phase-gradient in the direction of the shear (in DIC) or in the direction of the asymmetry (in AIDPC). Linear-gradients computed in two orthogonal directions are used to retrieve an image proportional to phase using frequency domain non-directional integration algorithm called spiral phase integration. 


\section{SWA4.pdf}
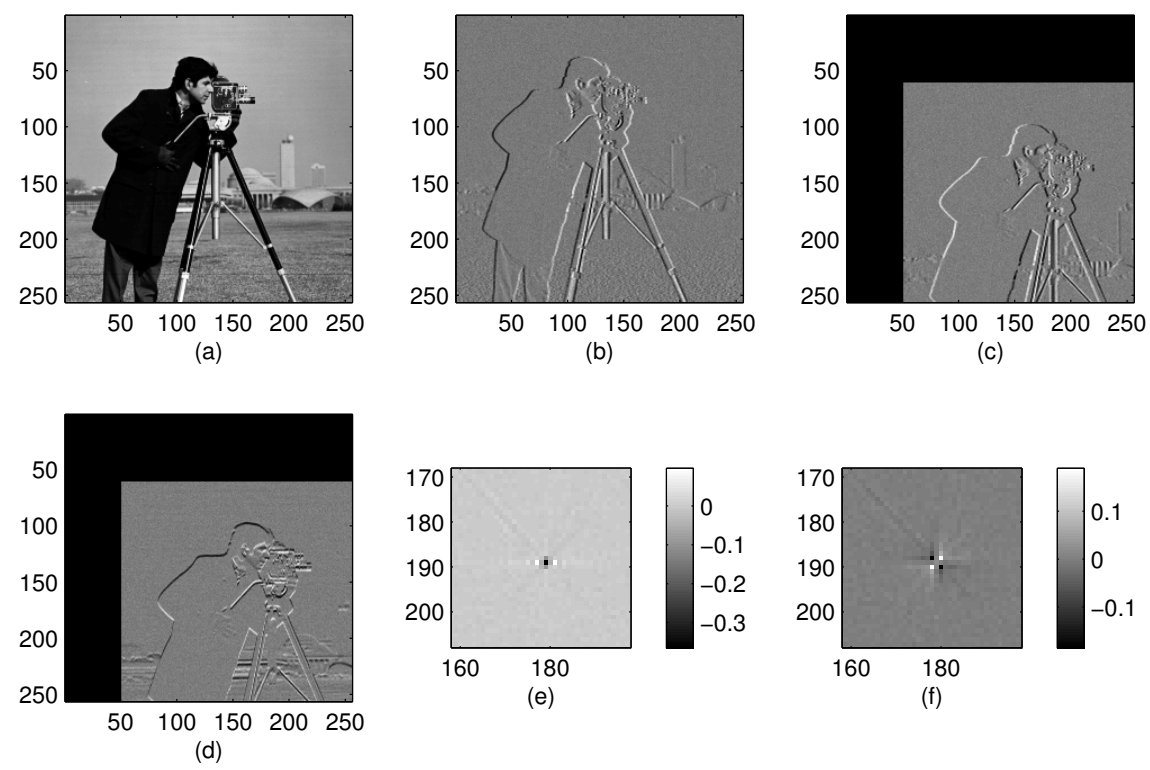

Fig. 1. Simulation of phase-correlation of $\mathrm{d} \phi / \mathrm{d} x$ with $-\mathrm{d} \phi / \mathrm{d} x$ and $\mathrm{d} \phi / \mathrm{d} y$ : (a) Original image of size 256X256, (b) gradient along $X$-direction, (c) gradient along -ve $X$-direction shifted by $(\Delta x, \Delta y)=(50,60)$, (d) gradient along $Y$-direction shifted by $(\Delta x, \Delta y)=(50,60)$, (e) phase-correlation of (b \& c), (f) phase-correlation of $(b \& d)$. Poisson noise with variance equal to the pixel-value was added to images (b, c, \& d). Images (e \& f) are zoomed to $40 \mathrm{X} 40$ pixels around the correlation peak. The negative correlation peak in (e) is situated at an offset $(50,60)$ from the center pixel $(129,129)$ of the image. The quad-peaks in (f) are centered around the same point.

In each direction, DIC requires registration of four images obtained at bias $0, \pi / 2, \pi$, and $-\pi / 2$; termed $I_{0}, I_{\pi / 2}, I_{\pi}$, and $I-\pi / 2$, respectively. AIDPC requires registration of only two images taken with left-half of the illumination aperture $\left(I_{L}\right)$ and right-half of the illumination aperture $\left(I_{R}\right) . I_{\pi / 2}$ and $I_{-\pi / 2}$ images of DIC measure phase-gradients with opposite contrast, so do $I_{L}$ and $I_{R}$ in AIDPC. To the first approximation, they represent $\mathrm{d} \phi / \mathrm{d} x$ and $-\mathrm{d} \phi / \mathrm{d} x$, mixed with amplitude information, $\phi(x, y)$ being the phase information to be retrieved. In other words, they represent gradients of phase in opposite directions. Since they are the same function but with opposite sign, the phase-correlation will be negative where both images are in register. Therefore, by finding position of minima of phase-correlation, we can find the relative shift.

Now, let us assume we need to register $I_{r}=\frac{\mathrm{d} \phi}{\mathrm{d} x}(x, y)$, and $I_{t}=\frac{\mathrm{d} \phi}{\mathrm{d} y}(x+\Delta x, y+\Delta y)$. Computing normalized cross-spectrum and phase-correlation according to eq. 1,

$$
\begin{aligned}
P\left(I_{t}, I_{r}\right) & =\mathcal{F}^{-1}\left[\frac{\left[i f_{x} \Phi\left(f_{x}, f_{y}\right) e^{i 2 \pi\left(f_{x} \Delta x+f_{y} \Delta y\right)}\right]\left[i f_{y} \Phi\left(f_{x}, f_{y}\right)\right]^{*}}{\left|f_{x}\right|\left|f_{y}\right|\left|\Phi\left(f_{x}, f_{y}\right)\right|^{2}}\right] \\
& =\mathcal{F}^{-1}\left[\operatorname{sign}\left(f_{x}\right) \operatorname{sign}\left(f_{y}\right) e^{i 2 \pi\left(f_{x} \Delta x+f_{y} \Delta y\right)}\right] \\
& =\frac{-1}{x y} \otimes \delta(x-\Delta x, y-\Delta y)=\frac{-1}{(x-\Delta x)(y-\Delta y)} .
\end{aligned}
$$

In the above, $\Phi\left(f_{x}, f_{y}\right)$ is the Fourier spectrum of the phase $\phi(x, y)$ and $\operatorname{sign}\left(f_{x}\right)=f_{x} /\left|f_{x}\right|$ is the signum function. It is seen that the phase-correlation in this case is a function that approaches positive and negative infinity around the point $(\Delta x, \Delta y)$. The phase-correlation appears as four peaks with positive and negative peaks placed diagonally with respect to each other.

Results of simulation that validate above derivation are shown in fig. 1. Predictions from above deriva- 


\section{SWA4.pdf}

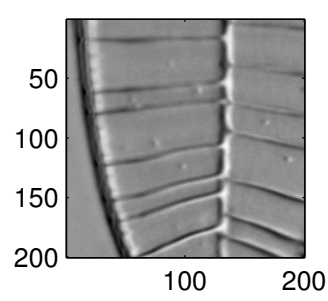

(a)

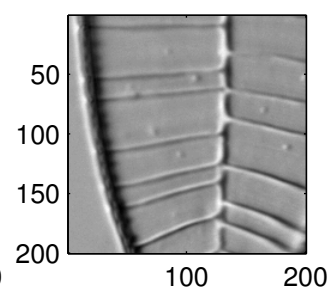

(b)

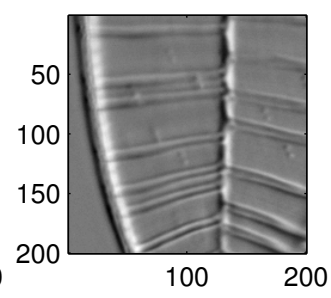

(c)

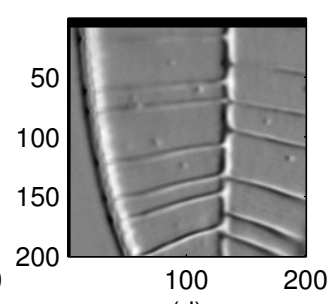

(d)

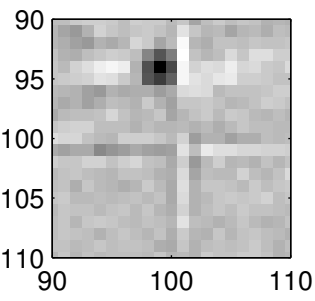

(e)

Fig. 2. Registration of experimentally acquired DIC images cropped to the size of 200X200 pixels. (a) Bias $\pi / 2$, (b) bias $-\pi / 2$, (c) difference of ( $\mathrm{a} \& \mathrm{~b}$ ) without registration shows duplication of features, (d) difference of $(\mathrm{a} \& \mathrm{~b})$ after registration produces an image with higher contrast, (e) Zoomed (20X20 pixels around center) phase-correlation map of (b) with respect to (a) shows the negative correlation peak at distance $(-2,-7)$ from the center.

tion are reproduced excellently in the discrete implementation of the algorithm. Experimental example of registration of DIC images taken with bias $\pi / 2$ and $-\pi / 2$ is shown in fig. 2 .

Note that DIC/AIDPC images do not exactly represent phase-gradient. Therefore, we employ forward image formation models $[4,10]$ to further refine phase-correlation criteria for registration of images which will be discussed in the presentation. Additionally, rotational mis-registration of two gradient images in orthogonal directions can be corrected using an extension of phase-correlation that transforms angular shifts into translation [8].

\section{References}

1. M. Pluta, Advanced Light Microscopy, vol. 2 Specialized Methods (PWN-Polish Scientific Publishers, Warszawa, 1989).

2. D. Hamilton and C. Sheppard, "Differential phase contrast in scanning optical microscopy," J. Microsc. 133, 27-39 (1984).

3. S. B. Mehta and C. J. R. Sheppard, "Asymmetric illumination based differential phase contrast (AIDPC) for full-field transmission imaging of phase information in biological specimens," (2008). Focus on Microscopy. URL: www.focusonmicroscopy.org/2008/PDF/089_Mehta.pdf.

4. S. B. Mehta and C. J. R. Sheppard, "Quantitative phase-gradient imaging at high resolution with asymmetric illumination based differential phase contrast (AIDPC)," Opt. Lett. 34 (2009). To appear.

5. M. R. Arnison, K. G. Larkin, C. J. R. Sheppard, N. I. Smith, and C. J. Cogswell, “Linear phase imaging using differential interference contrast microscopy," J. Microsc. 214, 7-12 (2004).

6. S. V. King, A. Libertun, R. Piestun, C. J. Cogswell, and C. Preza, "Quantitative phase microscopy through differential interference imaging," J. Biomed. Opt. 13, 024020 (2008).

7. C. D. Kuglin and D. C. Hines, “The phase correlation image alignment method," in "Proc. Int. Conf. on Cybernetics and Society," , vol. 4 (1975), vol. 4, pp. 163 - 165.

8. B. Zitova and J. Flusser, "Image registration methods: a survey," Image and vision computing 21, 9771000 (2003).

9. C. A. Glasbey and N. J. Martin, "Multimodal microscopy by digital image processing," J. Microsc. 181, 225-237 (1996).

10. S. B. Mehta and C. J. R. Sheppard, "Partially coherent image formation in differential interference contrast (DIC) microscope," Opt. Express 16, 19462-19479 (2008). 